\title{
Diseño de un controlador computarizado especializado para la granja experimental de la USCO
}

\section{Design of a specialized computerized controller for the experimental farm of the USCO}

\author{
Faiber Ignacio Robayo B. , Raúl Andrés Mosquera G. ${ }^{2}$ y Francisco Javier Cortes C. ${ }^{3}$
}

\begin{abstract}
Resumen
El presente trabajo se realiza en la granja experimental de la USCO (Universidad Surcolombiana) con el propósito de diseñar y construir un sistema de control computarizado y especializado para el sistema de riego, la cual se ubica en el perímetro rural del Juncal, jurisdicción del municipio de Palermo en el departamento del Huila, Colombia, con el fin de controlar adecuadamente el suministro de agua y ahorrar recursos de una forma más eficiente y menos compleja.
\end{abstract}

Para llevar a cabo esta tarea es necesario conocer los distintos tipos de riego que se aplican en el sector de estudio, las técnicas a utilizar, las variables necesarias para la aplicación de dichas técnicas, la maquinara y herramientas que se usan para el riego y posteriormente evaluar dichos factores e integrarlos para diseñar un instrumento que cumpla con la funcionalidad y con el factor académico e investigativo que caracterizan a la granja.

El resultado obtenido, es un prototipo especializado para el control de riego con múltiples funciones obtenidas a través del análisis de las necesidades que presenta la zona de aplicación. Este dispositivo es robusto, estable y permite ampliar su capacidad para adaptarse al terreno aplicado. Con este diseño, no solo se logra ahorrar en recursos y lograr mayor efectividad en cuanto al proceso de riego, sino que también se abre el camino para continuar con un trabajo interdisciplinar que impulse a la granja como principal fuente de investigación de la mano de los programas de ingeniería Electrónica e ingeniería Agrícola.

Palabras clave: Automatización; Control Computarizado; Programa; Sistema de riego; Adquisición de datos; Riego de presión.

\begin{abstract}
This work is carried out in the experimental farm of the USCO (Surcolombiana University) for the purpose of designing and building a computerized and specialized control for the irrigation system, which is located in the rural perimeter of Juncal, jurisdiction Palermo in the region of Huila, Colombia, in order to adequately control the water supply and save resources in a more efficient and less complex way.

To perform this task it was necessary to know the different types of irrigation systems applied in the field of study, the techniques to be used, the variables and tools used for irrigation in order to evaluate and integrate these factors in an instrument that meets the functionality and others academic and research factors of the farm.

The result is a specialized irrigation control with multiple functions obtained through the analysis of the needs presented by the area of application prototype. This device is robust, stable and able to expand their capacity to

1 - Magister en Ingeniería de Control. Docente Universidad Surcolombiana.Av. Pastrana Borrero carrera $1^{\text {a }}$ Neiva. E-mail: faiber.robayo@usco.edu.co

2- Ingeniero Electrónico. Universidad Surcolombiana. Av. Pastrana Borrero carrera $1^{\mathrm{a}}$ Neiva. E-mail: raulandresmg@gmail.com

3 - Ingeniero Electrónico. Universidad Surcolombiana. Av. Pastrana Borrero carrera 1ª Neiva. E-mail: fjcc2007@hotmail.com
\end{abstract}


adapt to the applied field. With this design, not only saving resources and greater effectiveness in the process of irrigation are achieved, but also opens the way for further interdisciplinary work that encourages farm as the main source of research at the hands of the programs Agricultural engineering and Electronics engineering.

Keywords: Automation; Computerized Control; program; irrigation system; acquisition; irrigation pressure.

\section{Introducción}

La Universidad Surcolombiana cuenta con una granja experimental ubicada en el perímetro rural del Juncal, jurisdicción del municipio de Palermo en el departamento del Huila, la cual es utilizada para la aplicación, mejoramiento y desarrollo de técnicas de riego (Santos et al., 2010), y a su vez para medir el impacto de la agricultura en todos los ámbitos que afectan a la sociedad, tales como el ambiente en el que se aplica, la eficiencia en el manejo de los recursos, la productividad obtenida, la relación costo/beneficio deseada y en general el aporte a la sociedad como resultado de la labor realizada según los estudios y normatividades establecidas por el Ministerio de Agricultura de Colombia (Condiza et al., 1998).

El suministro de agua y nutrientes, la geografía del área y el clima, son el objeto de interés para el agricultor, por lo tanto se hace necesario tener un sistema adecuado para la adquisición de la información relacionada a las variables anteriormente mencionadas, con el fin de ser analizadas por parte del productor que mediante el conocimiento, manejo y experiencia en la actividad agrícola reconoce y determina las necesidades que el cultivo requiere ya que es imposible confiar en un sistema por alto grado de tecnificación o automatizado que este tenga, además de las acciones que el sistema debe ejecutar, todo esto con el fin de reducir los errores humanos en el manejo y administración de las tareas para así proporcionar datos confiables y de relevancia para la comunidad agrícola (Soler, 2011), de esta manera la integración de los datos obtenidos con la toma de decisiones por parte de los especialistas en el área de acción es muy importante, debido a que "la participación activa del productor en la planificación y establecimiento del diseño del riego" es esencial para la productividad del terreno (Carrazón, 2007).

Las necesidades estructurales como el diseño de los sistemas de riego, los métodos de irrigación, el número de sectores de riego y los mecanismos de acción en la ductería son las características físicas a tener en cuenta a la hora de la integración del área de trabajo en relación con el sistema que gobierna la administración de las tareas a realizar, de esta manera su determinación y selección aporta las bases en el desarrollo de los dispositivos hardware a implementarse. Adicionalmente es importante el estudio y determinación de los componentes externos al equipo tales como sensores, contactores y los diferentes tipos y estándares manejados por las electroválvulas que corresponden a la salida final del sistema (Wahl, 2013).

Este proyecto abre camino para el desarrollo y aplicación de nuevas tecnologías en la agricultura, y sienta las bases para el concepto de agricultura de precisión, que aplica sistemas de Redes Integradas de Datos, posicionamiento global, monitoreo y sensado de variables con el objetivo de la mejor relación costo beneficio en la productividad del cultivo (Bragachini etal.,2005).

\section{Metodología}

El diseño de un sistema de control computarizado especializado para la granja de la USCO y la construcción de un prototipo para probar su funcionamiento y verificar que su aplicación da solución a las necesidades presentes en la zona de aplicación, fue dividido en las fases descritas a continuación.

\subsection{Especificaciones $y$ diseño del prototipo hardware del controlador}

A fecha de 2014, la granja experimental cuenta con 9 sectores de riego en los cuales existen 11 válvulas en total (válvulas activas), a estas 11 válvulas se les suman las válvulas de control de la casa de máquinas, que son 3, correspondientes al mecanismo de lavado de filtros y 3 más, que son las que controlan el paso de agua del tanque de cebado a la bomba del sistema de riego por aspersión.

En cuanto a las variables de campo a medir el controlador debe tener la capacidad de conectarse a cualquier tipo de sensor según las normas técnicas estándares, ya que el especialista en interpretación de datos agrícolas puede necesitar una gran cantidad de 
variables como $\mathrm{pH}$, humedad, niveles de oxígeno, luz, etc., para diagnosticar y valorar de la mejor forma las condiciones del cultivo. La extensión del terreno a controlar juega un papel muy importante en el diseño, ya que la distancia que existe entre el sistema de control central y los periféricos es un problema a superar, según la geografía del terreno, se implementan sistemas de comunicación acordes con las características de la zona para buscar un mejor desempeño $\mathrm{y}$ rendimiento del controlador.

Para la granja experimental de la USCO se tienen dos distancias que influyen directamente en el diseño del control de riego, el controlador idealmente estará ubicado en el hangar o en la casa situada al lado del hangar por su fácil acceso, por lo tanto la estación de bombeo se ubica a 250 metros aproximadamente de la estructura principal, esta es la primera distancia a superar para permitir la integración del encendido de las bombas; la segunda distancia es desde la estación central donde se ubica el controlador hasta las válvulas que deben ser controladas. Otro aspecto de gran importancia que se tiene en cuenta para el diseño del sistema de control son los ciclos de riego que corresponden a periodos de tiempo en los que cada sector de riego recibe la atención de cada etapa del sistema desarrollado, según la madurez del cultivo y la estación climática en la que se encuentra se determina dicha duración y periodicidad por parte del equipo encargado de esta labor. Una vez este itinerario ha sido establecido por dichos funcionarios, estos ciclos de riego deben ser ejecutados y supervisados para así verificar la eficiencia y rendimiento en razón al costo/beneficio aportado al cultivo.

El controlador a nivel de hardware está compuesto por 4 tarjetas electrónicas, las cuales son: tarjeta principal que posee 2 microcontroladores $18 \mathrm{f} 4550$, una memoria EEPROM (24LC512), un reloj digital (DS1307) y una memoria micro SD de $2 \mathrm{~GB}$ con sus respectivas configuraciones, esta tarjeta se encarga de recibir las configuraciones elaboradas en la interfaz gráfica de usuario, almacena las rutinas de riego en la memoria EEPROM y las activa dependiendo de la hora predeterminada, también almacena y lee las variables desde la tarjeta de acople de transductores, activa y desactiva las electroválvulas enviado la orden respectiva a la tarjeta electrónica que corresponda y lleva una bitácora de las actividades realizadas por el controlador almacenado en la memoria micro SD un archivo .txt con dichas actividades a la hora y fecha ejecutadas. Esta configuración puede observarse en la Figura 1.

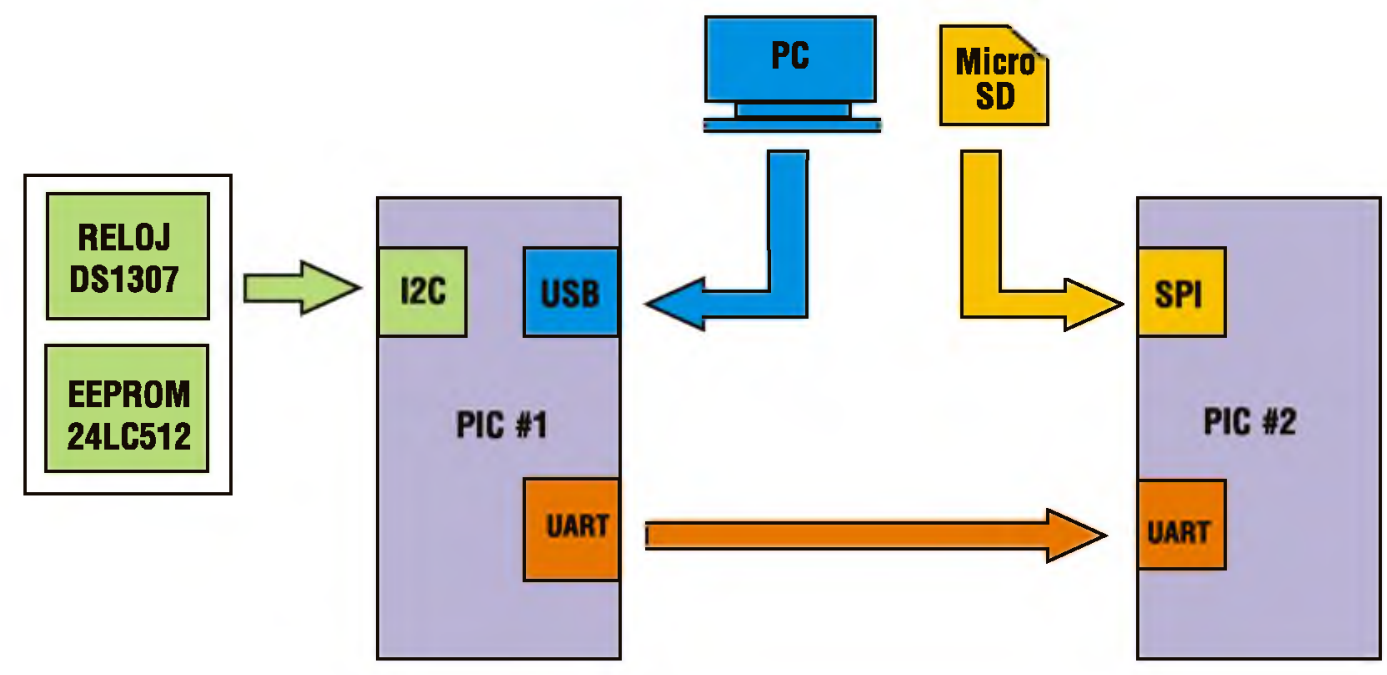

Figura 1. Comunicación interna de la tarjeta electrónica principal

La tarjeta electrónica número 2 se encarga de acoplar los transductores a las entradas del módulo conversor analógico-digital de la tarjeta número 1 para que las señales se lean de acuerdo a un formato específico y hacer que esta lectura sea lo más exacta posible. Para realizar el acople de impedancias entre los transductores y las entradas de los módulos, se utiliza un circuito integrado LM358 en configuración de ganancia unitaria y un interruptor de perilla para realizar la conmutación entre los dos formatos de transmisión de la señal de datos soportados por el controlador las cuales son de 4 a 20 mili amperios (mA) y de 0 a 5 voltios (V). 
La tarjeta encargada de activar y desactivar las electroválvulas es la tarjeta electrónica número 3, la cual está compuesta por 20 relés, de los cuales cada uno de ellos asiste una electroválvula específica, la información es recibida por un conector de 22 pines, unido a un bus de datos el cual se conecta del otro extremo a la tarjeta principal, específicamente a los puertos $\mathrm{B}, \mathrm{C}, \mathrm{D}$ y E del microcontrolador número 2 de la tarjeta principal, el bus de datos posee 22 pines, 20 para activar los relés y 2 para tierra común.

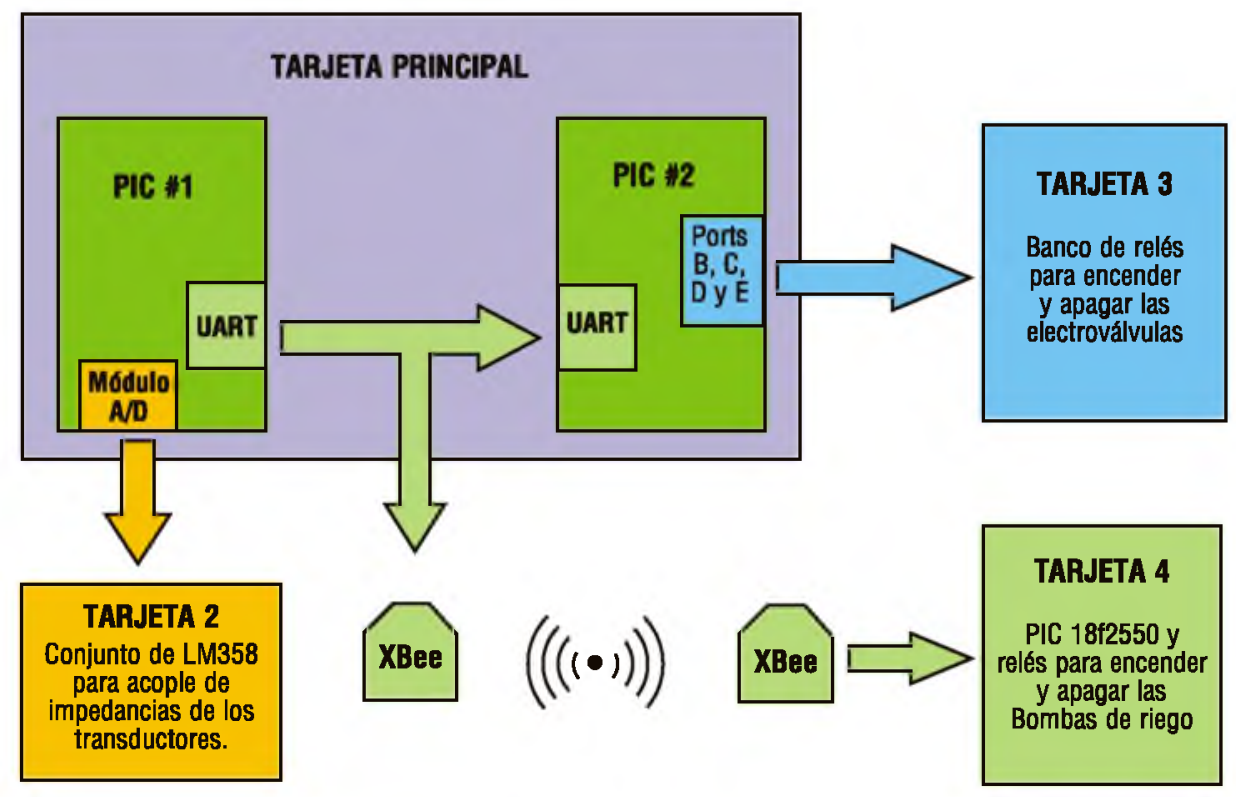

Figura 2. Conexiones eléctricas entre las tarjetas que conforman el controlador

La distancia entre la casa de bombeo y el terreno de distribución de las electroválvulas es bastante larga (aproximadamente 500 metros), por lo tanto y como se especifica anteriormente se busca un sitio intermedio entre los dos para ubicar el centro de control, este sitio está ubicado cerca o dentro del hangar (aproximadamente a 250 metros de la casa de bombeo) donde se guarda la maquinaria para trabajar la tierra, es por esta razón que la transmisión entre el controlador y la activación de las bombas de riego se hace por medios inalámbricos. Para llevar a cabo esta tarea es necesario recibir y procesar la señal transmitida por el módulo transceptor XBee conectado al microcontrolador número 1 de la tarjeta electrónica principal, la tarjeta encargada de recibir esta información es la tarjeta electrónica número 4 que está compuesta por otro módulo transceptor XBee conectado a un microcontrolador $18 \mathrm{~F} 2550$ por medio del protocolo de comunicación UART (Universal Asynchronous Receiver Transmitter), y 6 relés encargados de activar y desactivar las bombas de riego, lavado de filtro y cebado de la bomba del sistema de riego por aspersión.

La conexión de las tarjetas electrónicas se encuentra descrita en la Figura 2, de acuerdo a las especifica- ciones de la bomba de riego por gravedad que es la más grande y la que mayor suministro de energía necesita. Los relés utilizados para la activación de las bombas de riego no soportan la corriente necesaria para el arranque y funcionamiento de dichos motores por lo tanto se hace uso de la etapa de potencia que posee las instalaciones de la casa de bombeo. La tarjeta 4 corresponde a la etapa de activación del sistema de maniobra y control del suministro de energía a los motores de las bombas, esta última labor es realizada por relés de tipo industrial llamados contactores (Aparatos de maniobra). En relación a lo anterior el circuito queda formado como se puede apreciar en la Figura 3.

Las salidas del controlador suministran niveles de 110 vol o 220 vol (dependiendo de la capacidad del relé, de la fuente que suministra la energía y de los niveles de activación del contactor) que alimentan las bobinas del contactor, estas a su vez activan el electroimán y desplazan el mecanismo móvil para unir los contactos de cada extremo los cuales por un lado están conectados a las líneas de alta tensión o en su defecto transformador y los otros terminales se encuentra unidos a la Bomba eléctrica produciendo el cierre del circuito y encendiendo el dispositivo. 


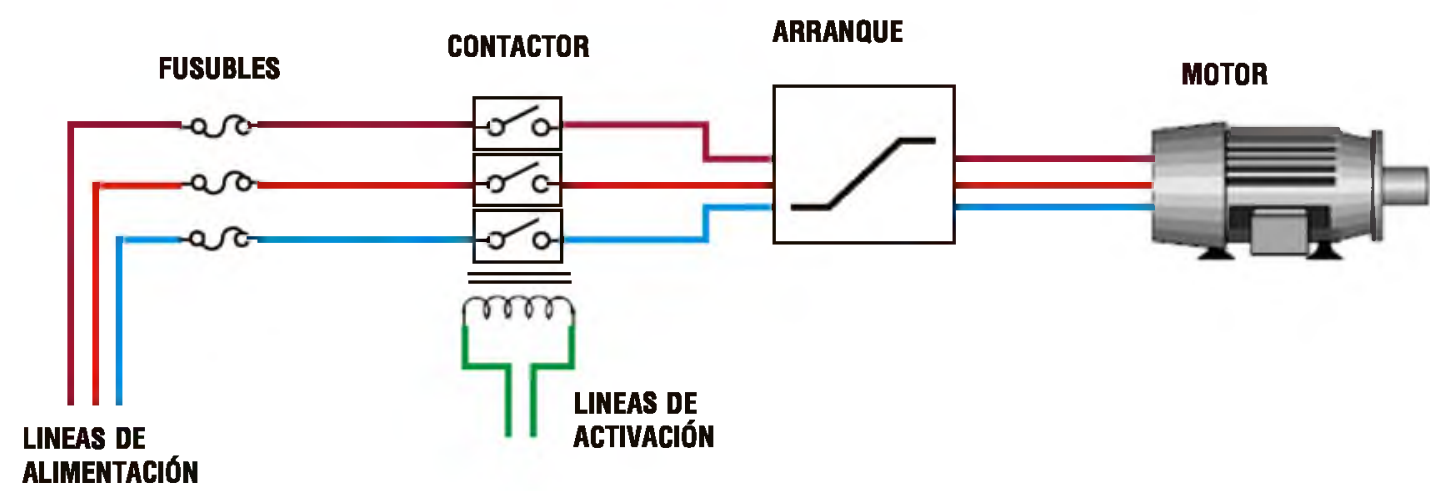

Figura 3. Circuito de alimentación de las bombas eléctricas de riego

\subsection{Diseño del software como medio de programación y/o configuración del controlador de riego}

Para organizar de la mejor forma las actividades a realizar por el controlador y lograr una interfaz gráfica amigable con el usuario el programa que configura el controlador se divide en 4 partes fundamentales las cuales se ubican cada una de ellas en una pestaña. La primera pestaña es llamada "Oficina" y es la encargada de inicializar el reloj del controlador y generar un reporte de la programación deseada en un archivo con formato .doc; esta función tiene el objetivo de servir como soporte de las configuraciones realizadas al controlador y llevar la información de riego de forma organizada y automática para posteriores análisis (Figura 4).

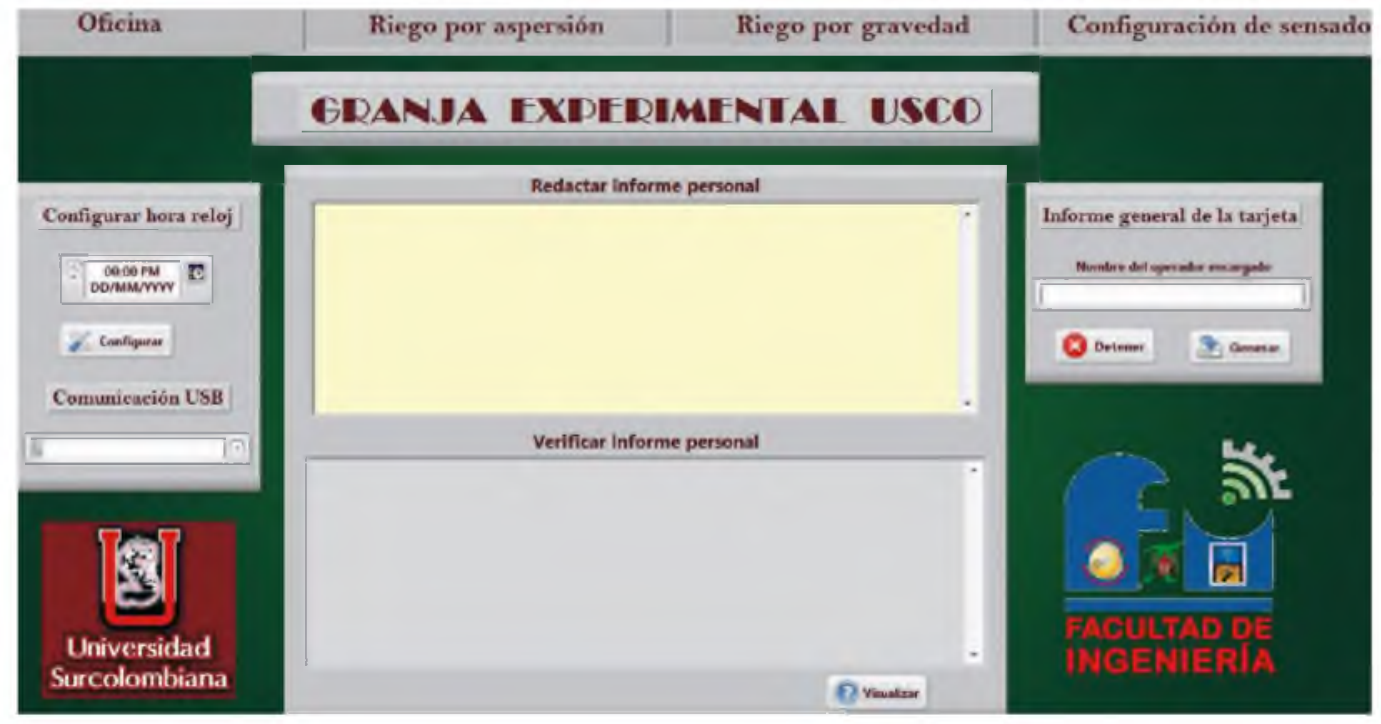

Figura 4. Pestaña No. 1 "Oficina"

La segunda pestaña llamada "Riego por aspersión" (Figura 5), como su nombre lo indica es utilizada para programar los eventos y turnos de riego para el sector irrigado por el método de aspersión. Esta pestaña cuenta con múltiples funciones entre las cuales se destacan: lavado de filtro, turnos de riego dentro de un evento para conmutar los sectores de riego, eliminación de un evento completo de riego y borrado de todas las actividades de riego programadas. A través del botón de "llenar tabla" podemos observar en tiempo real qué actividades o turnos de riego están gravados en la memoria del controlador y por consiguiente se van a ejecutar. Para programar el fin e inicio de evento y turno la aplicación cuenta con controles horarios y casillas de incremento en horas y minutos; el lavado de filtro y las válvulas a activar posee un espacio individual dentro de la pestaña para organizar la información y facilitar el manejo del usuario. 


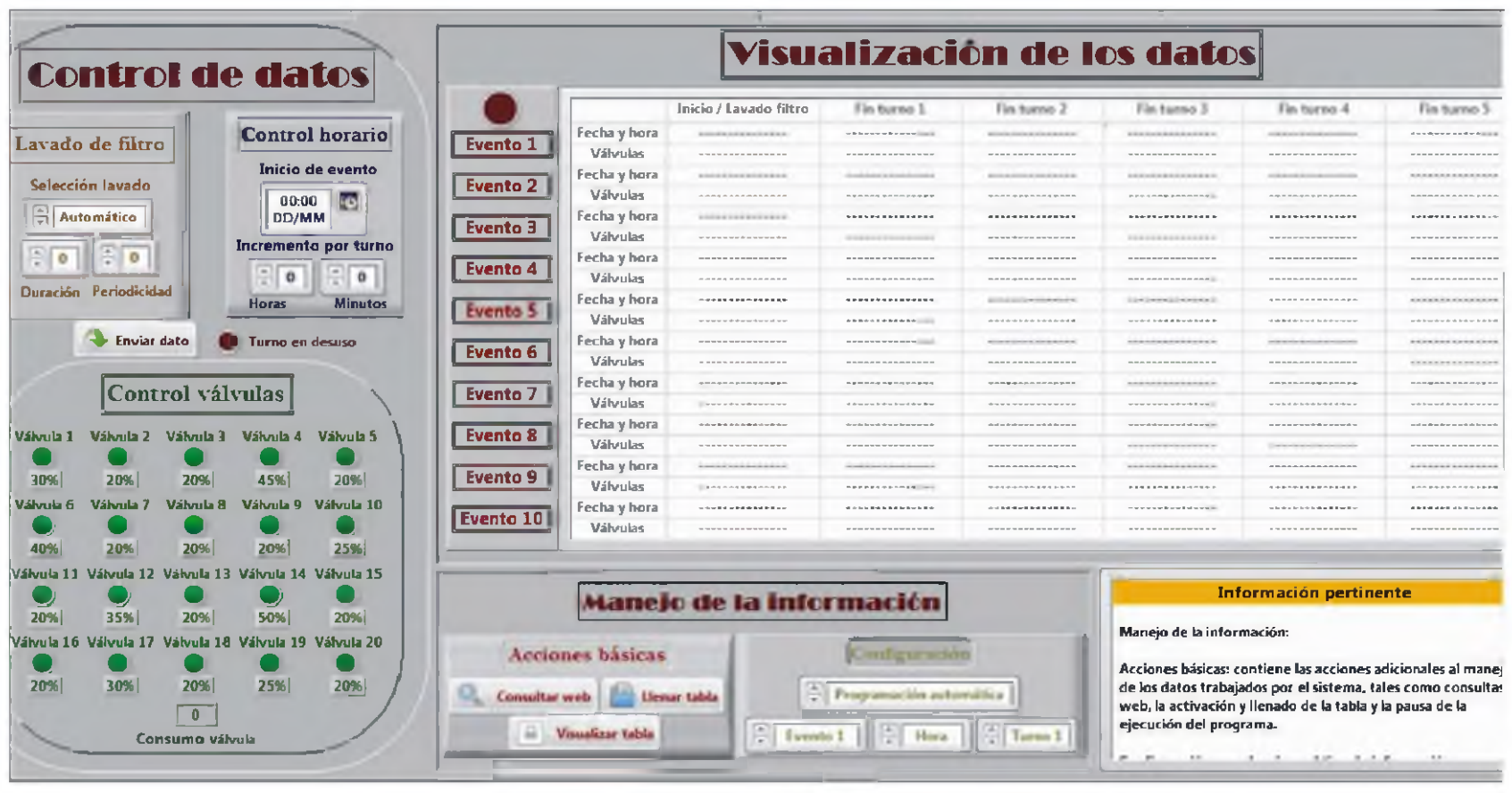

Figura 5. Pestaña No. 2 "Riego por aspersión"

La pestaña "Riego por gravedad" se divide en dos partes muy importantes, la primera es la función característica de esta pestaña la cual se encarga de la programación de un cronograma de 28 eventos de riego los cuales no tienen mayor complejidad, simplemente una fecha y hora de inicio de evento y una fecha y hora de fin de evento de riego. Además de la anterior función esta parte de la pestaña cuenta con la corrección de un evento y el borrado del total de los eventos de riego (equivalente al borrado o puesta a cero de la tabla que simboliza los registros o espacios de programación del riego por gravedad). La segunda parte de la pestaña Riego por gravedad se encarga de leer los registros de la memoria en donde se han almacenado los datos de la lectura de los transductores, además, borrar los registros e inicializar la hora de guardado de la información o los valores de las variables deseadas (Figura 6).

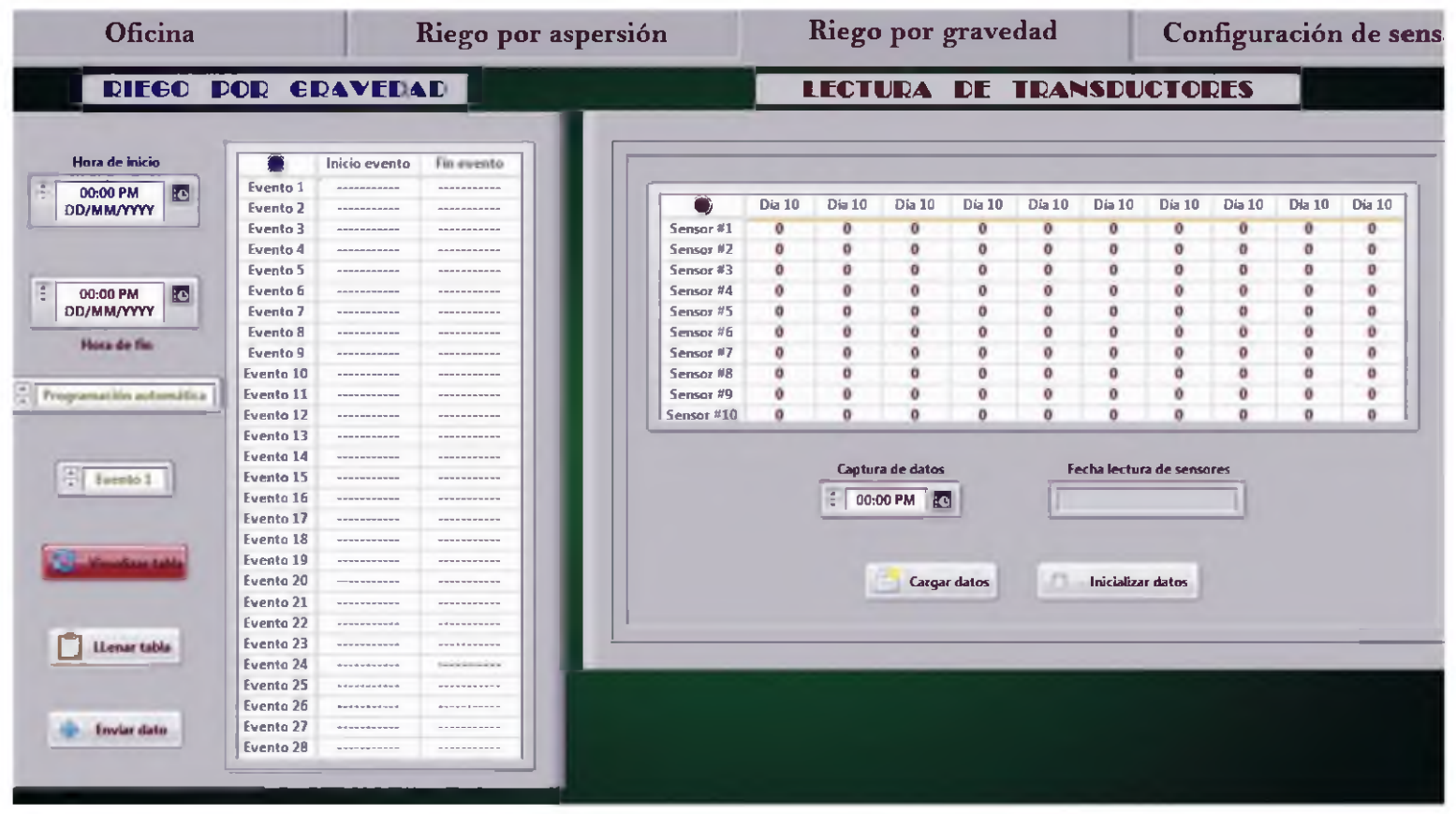

Figura 6. Pestaña No. 3 "Riego por gravedad" 
Por último y como complemento de la tercera pestaña, la pestaña número 4 se encarga de brindar al usuario las herramientas necesarias para establecer las ecuaciones de cada uno de los 10 canales de adquisición de las variables para procesar los datos, entonces el modelamiento de las curvas de respuesta de los transductores utilizados se realizan estableciendo las variables de entrada, las variables de salida y las ecuaciones que describen dichas curvas de respuestas. Además, se puede seleccionar para cada canal el formato de recepción de datos de 4 a 20 mAy de 0 a 5 V, (Figura 7).

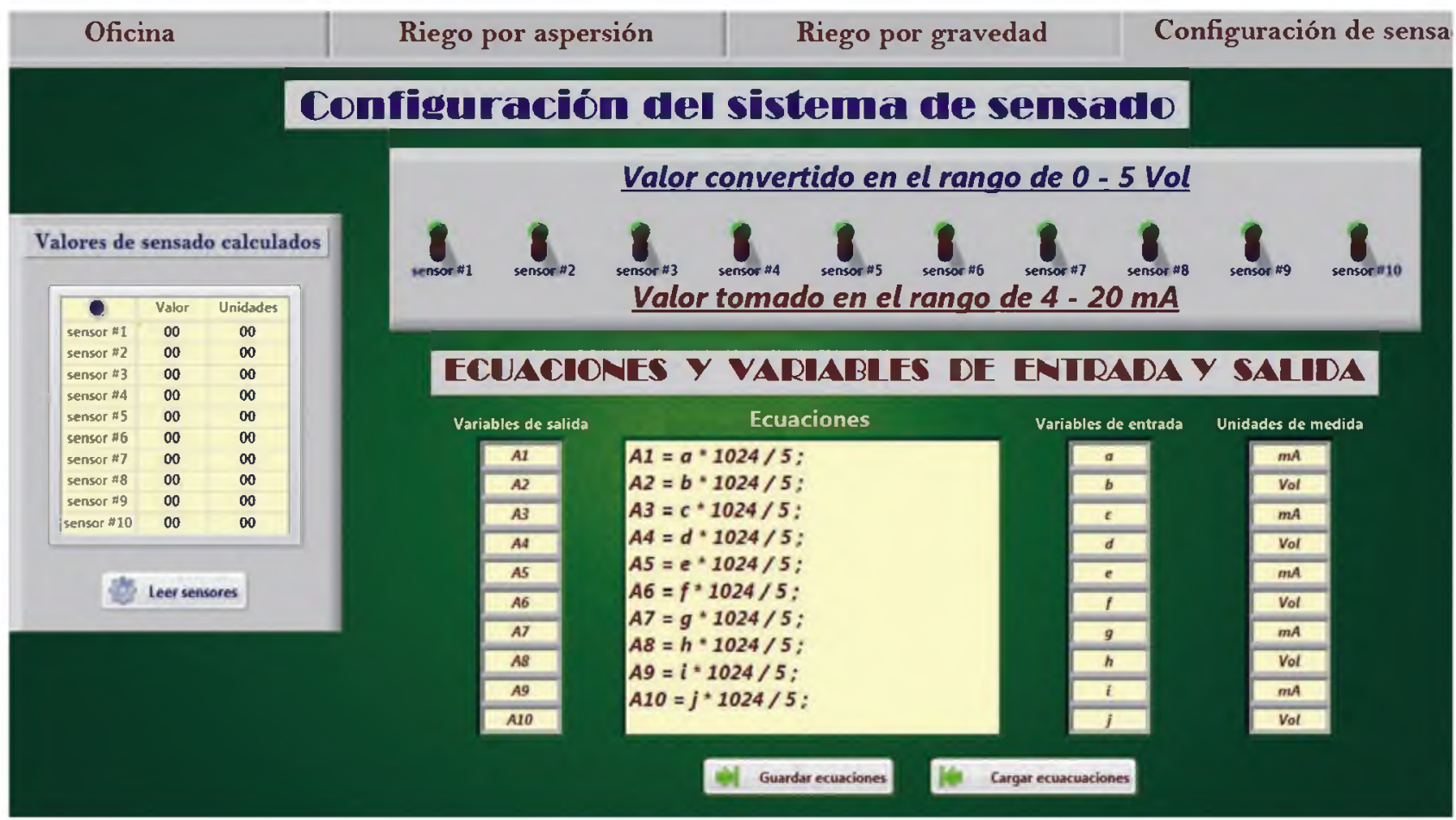

Figura 7. Pestaña No. 4 "Configuración de sensado"

\subsection{Canales de adquisición de datos}

Para realizar el acople de la señal de los transductores con las entradas de los pines de conversión analógicodigital del microcontrolador utilizados para la adquisición de los datos contamos con una tarjeta que posee un circuito LM358 el cual contiene dos circuitos integrados dentro del mismo encapsulado (Low Power Dual), cada amplificador operacional en configuración seguidor unitario se utiliza para adecuación de la señal de una entrada de adquisición de datos, a la entrada del A/D (Analógico/Digital) del microcontrolador.

La configuración seguidor unitario se utiliza por las siguientes razones: voltaje de salida igual al voltaje de entrada (Vout $=$ Vin) y resistencia de entrada alta, por lo tanto extrae corriente despreciable de una fuente de señal. Esta característica es útil cuando se quiere convertir corriente en voltaje a través de una resistencia de precisión (4-20 $\mathrm{mA}$ a $0-5 \mathrm{~V}$ ) y aprovechando las características generales de un amplificador operacional como son la alta impedancia de entrada (MS) y la baja impedancia de salida se logra eliminar efectos de carga, para así lograr adaptar impedancias de diferentes etapas, que según las características anteriormente descritas son útiles para la lectura de niveles de voltaje, disminuyendo los errores en las lecturas por resistencia de carga (Coughlin, Driscoll, 1993).

\section{Resultados}

El sistema de control enciende y apaga dos motores de bombeo, uno para el riego por gravedad y el otro para el riego por aspersión, el cual posee un sistema de filtrado que tiene como función eliminar los residuos contenidos en el fluido que circula por la ductería, el cual se debe limpiar periódicamente, función que también es realizada por el controlador diseñado. Además de las bombas controladas el sistema realiza la apertura y el cierre de las electroválvulas que 
determinan el riego de los diferentes sectores existentes en las instalaciones de trabajo, además de contar con 10 canales de adquisición de datos los cuales se pueden configurar para el manejo de dos diferentes estándares manejados por los transductores según los niveles de señal de transmisión de la información, correspondientes al estándar de 4 a 20 $\mathrm{mA}$ o de 0 a $5 \mathrm{~V}$.
El resultado obtenido, es un prototipo para el control de riego con múltiples funciones obtenidas a través del análisis de las necesidades que presentaba la zona de aplicación (granja experimental USCO). Este dispositivo es robusto, estable, con perspectiva de crecimiento en el terreno aplicado, (Figura 8).

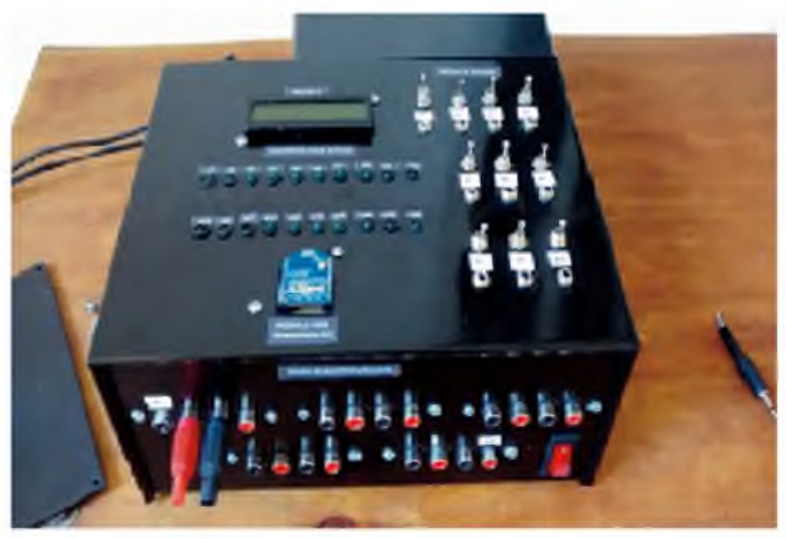

Figura 8. Controlador computarizado real

Para comprender mejor el dispositivo diseñado se presenta la siguiente tabla de especificaciones técnicas:

Tabla 1. Características técnicas del controlador diseñado

\begin{tabular}{ll}
\hline Características & Descripción \\
\hline Número de válvulas a controlar & $\begin{array}{l}20 \text { electroválvulas de riego por aspersión, } 3 \text { del } \\
\text { sistema de cebado, 3 del sistema de lavado de filtros }\end{array}$ \\
\hline Número de canales analógicos & 10 canales de adquisición de datos \\
\hline Entradas & Estándar 4-20 mA y 0-5 vol \\
\hline Salidas & 24 VAC / 12 VAC a 250 mA \\
\hline Número de bombas a controlar & $\begin{array}{l}1 \text { bomba de riego por gravedad y } 1 \text { bomba de riego por } \\
\text { aspersión }\end{array}$ \\
\hline Potencia consumida & Máxima potencia consumida $=122.5$ watts \\
\hline Reporte de seguridad & $\begin{array}{l}\text { Copia de las actividades realizadas por el controlador } \\
\text { en una micro SD generada en un archivo .txt }\end{array}$ \\
\hline Sistema de comunicación híbrido & $\begin{array}{l}\text { Comunicación con las electroválvulas mediante } \\
\text { cableado de cobre y comunicación con la casa de } \\
\text { bombeo por radiofrecuencia }\end{array}$ \\
\hline
\end{tabular}

Actualmente existen diversos controladores de riego que se enfocan en solucionar algunas necesidades, uno de los más utilizados es el controlador modelo 800248 , a continuación se presentan las comparaciones entre los dos controladores: 
Tabla 2. Tabla comparativa entre controlador comercial y controlador diseñado

\begin{tabular}{|c|c|}
\hline Controlador Modelo 800248 & Controlador Granja Experimental USCO \\
\hline $\begin{array}{l}\text { Controla el funcionamiento de } 1 \text { a } 24 \text { válvulas, más } \\
\text { válvula principal o bomba }\end{array}$ & $\begin{array}{l}20 \text { electroválvulas de riego por aspersión, } 3 \text { del sistema } \\
\text { de cebado, } 3 \text { del sistema de lavado de filtros, } 1 \text { bomba de } \\
\text { riego por gravedad y } 1 \text { bomba de riego por aspersión }\end{array}$ \\
\hline Opera válvulas eléctricas 24VAC & Opera válvulas eléctricas $24 \mathrm{VAC}$ o $12 \mathrm{VAC}$ \\
\hline $\begin{array}{l}\text { Ciclo de riego desde una vez al día hasta una vez cada } \\
30 \text { días }\end{array}$ & $\begin{array}{l}\text { Para el riego por gravedad el sistema cuenta con } 28 \\
\text { eventos de riego. } \\
\text { Los ciclos para el riego por aspersión se organizan en } 10 \\
\text { eventos y los eventos se dividen en } 5 \text { turnos de riego. } \\
\text { Cuenta con un calendario que permite seleccionar } \\
\text { cualquier fecha de activación para los eventos }\end{array}$ \\
\hline Duración de riego desde 1 minuto a 9 horas & Los turnos pueden ir desde 1 minuto hasta 24 horas \\
\hline $\begin{array}{l}\text { Copia de seguridad (backup) en memoria del } \\
\text { programa por } 20 \text { años }\end{array}$ & $\begin{array}{l}\text { Copia de las actividades realizadas por el controlador en } \\
\text { una micro SD, en un archivo .txt }\end{array}$ \\
\hline $\begin{array}{l}\text { Batería de 9V para mantenimiento de la hora hasta } 90 \\
\text { días (no incluida) }\end{array}$ & $\begin{array}{l}\text { Batería de } 3 V \text { de litio para mantenimiento de la hora } \\
\text { hasta } 1 \text { año(incluida) }\end{array}$ \\
\hline $\begin{array}{l}\text { Entrada de sensores de lluvia, temperatura, o } \\
\text { humedad }\end{array}$ & $\begin{array}{l}10 \text { canales de adquisición de datos las entradas pueden } \\
\text { ser 4-20 mA o 0-5 vol, con entradas en configuración } \\
\text { seguidor unitario (alta impedancia de entrada) }\end{array}$ \\
\hline
\end{tabular}

La programación de la interfaz de usuario, prevé situaciones de error humano en la configuración de las actividades de riego del controlador tales como:

- El envío de posibles datos erróneos correspondientes al itinerario programado y las electroválvulas que se deben accionar en cada uno de los turnos en uso, esto se previene mediante un sistema de seguros que impide la interacción del usuario con cualquier conjunto de controles que no deban usarse según el modo en el que se encuentra configurado el sistema (Programación automática, Lavado de filtro, Programación manual, Eliminar evento, Limpiar tabla) con el fin de evitar el guardado de datos de tipo no correspondientes en cada sección de la memoria, además de un sistema interno que se encarga de calcular los valores adecuados del consumo de las válvulas accionadas y de los tiempos de acción para la ejecución del lavado de filtro del sistema de riego.

- La posible modificación del itinerario de trabajo del sistema por parte de personas ajenas a esta labor o errores de los operarios en los cuales se altere el calendario y los sectores de riego activos configurados por el personal correspondiente. Esto se previene mediante la adición de un sistema de verificación integrado al controlador el cual enlaza la memoria del dispositivo con la aplicación software lo cual permite la visualización de toda la información programada en el sistema permitiendo generar en un archivo en formato .doc el cual contiene el itinerario a ejecutarse por el controlador, además se incluye un reporte histórico que permite verificar posibles ejecuciones afectadas por cambios imprevistos.

\section{Conclusiones}

El conjunto que forma el sistema de control y supervisión habilita la posibilidad a futuro de nuevas expansiones de ser necesario. La configuración interna que conforma el software diseñado permite de una forma muy sencilla integrar nuevos sectores de riego, eventos y turnos para ser programados y permite expandir el número máximo de válvulas a activar de forma simultánea, por otra parte el hardware que conforma el controlador permite la adición de tarjetas destinadas a controlar nuevos sectores apoyadas por la comunicación serial usada.

El sistema tiene como finalidad no solo el control y la administración del riego sino también la capacidad de aportar a los docentes y/o investigadores una herramienta versátil para impartir sus clases y mostrar la operatividad del sistema utilizado, así mismo dar claridad de la función que cumple cada una de las variables encargadas de la operación de este tipo de control.

El controlador diseñado cumple con las condiciones específicas de instalación requeridas en la infraestructura de la granja experimental de la USCO. Este sistema está dividido en dos partes intercomu- 
nicadas las cuales cumplen con distintas funciones en el manejo del riego, una es la parte que maneja la etapa de sensores y la activación de los sectores de riego mientras la otra se encarga de la activación de las bombas y el lavado de filtro. Para la sección de riego por aspersión estas tarjetas están intercomunicadas por medio de dos módulos transceptores que se encarga de sincronizar las diferentes funciones que se deben cumplir acoplándolas de manera inalámbrica, este tipo de conexión fue necesario implementarse debido a las condiciones presentes en el área de operación.

El diseño de la interfaz que conforma el software desarrollado permite al usuario diseñar y programar los diversos eventos de riego necesarios en la granja experimental de la USCO de una forma versátil y amigable permitiendo su fácil manejo y fluidez al momento de su utilización, ya que la distribución de toda la información está integrada en una sola tabla para su visualización, permitiendo enviar y recibir los datos programados en tiempo real para que puedan ser consultados y/o modificados por el administrador en cualquier momento; además se puede generar un reporte de la información en un formato estándar creado en un documento de Microsoft Word para su impresión y que puede ser utilizado como una referencia del itinerario de riego programado.

\section{Referencias Bibliográficas}

1. Aparatos de maniobra Contactores y relés de sobrecarga, 2010. Arrancadores de motor con contactores. Catálogo técnico, División Control y Automatización, pp. 5/2-5/33. [En línea]. Disponible en http://www.abb.com.co. Consultada el 05 de septiembre de 2013 de ABB.
2. Bragachini, M., Méndez, A., Scaramuzza, F., Proietti, F., 2005. Proyecto Agricultura de Precisión. Instituto Nacional de Tecnología Agropecuaria (INTA), Estación experimental agropecuaria Manfredi, Córdoba Argentina. pp. 1-11.

3. Carrazón, J., 2007. Manual práctico para el diseño de sistemas de minirriego. Programa especial para la seguridad alimentaria (PESA), Organización de las Naciones Unidas para la agricultura y la alimentación (FAO), Honduras. pp. 15-104.

4. Condiza, C.A., Talero, A.A., González, F., 1998. Agricultura sostenible. Programa nacional de transferencia de tecnología agropecuaria (PRONATTA), ministerio de agricultura, república de Colombia: Litografía Géminis LTDA. pp. 8-16.

5. Coughlin, R.F., Driscoll, F.F., 1993. Amplificadores operacionales y circuitos integrados lineales, México: Prentice Hall Hispanoamericana S.A. $4^{\mathrm{a}}$ Ed., pp. 43144.

6. LM158/LM258/LM358/LM290. Low Power Dual Operational Amplifiers, 2013. [En línea]. Disponible en http://www.ti.com/lit/ds/symlink/lm158-n.pdf . Consultada el 14 de abril de 2013 de Texas Instruments.

7. López, E., 2008. Ingeniería en microcontroladores protocolo USB (Universal Serial Bus), i-micro, México D.F.pp. 1-11.

8. Santos, L., de Juan Valero, J.A., Picornell, M.R., Tarjuelo, J.M., 2010. El riego y sus tecnologías. Centro Regional de Estudios del Agua (CREA), Universidad de Castilla-La Mancha (UCLM), Albacete España. ,pp. 87-224.

9. Soler, M., 2011. Curso de perfeccionamiento en automatización y telecontrol de sistemas de riego ( $2^{\mathrm{a}}$ edición), automatismos empleados para la gestión de la fertirrigación en parcela $y$ en invernaderos. Agrosolmen, Murcia España. $3^{\mathrm{a}} \mathrm{Ed}$., pp. 1-25.

10. Wahl, P., 2013. Libro blanco Válvula de corredera válvula de asiento, Una comparación técnica para diversas electroválvulas, Festo AG \& Co. KG. pp. 1-5. 